\title{
Evolution of Endovascular Therapy in Acute Stroke: Implications of Device Development
}

Adithya Balasubramaian, Peter Mitchell, Richard Dowling, Bernard Yan

Comprehensive Stroke Centre, Royal Melbourne Hospital, Parkville, Victoria, Australia

Journal of Stroke 2015;17(2):127-137 http://dx.doi.org/10.5853/jos.2015.17.2.127

The first author's name was misprinted. The name should be corrected as follows.

\section{Corrected Author's Name}

Adithya Balasubramanian, Peter Mitchell, Richard Dowling, Bernard Yan 